\title{
A comparative profitability analysis of transcatheter versus surgical aortic valve replacement in a high-volume French hospital
}

François Huchet ${ }^{1 *}$ (D), Jacques Chan-Peng ${ }^{1}$, Fanny d'Acremont ${ }^{2}$, Patrice Guerin ${ }^{1}$, Gael Grimandi ${ }^{2}$, Jean-Christian Roussel ${ }^{3}$, Julien Plessis ${ }^{1}$, Vincent Letocart ${ }^{1}$, Thomas Senage ${ }^{3}$ and Thibaut Manigold ${ }^{1}$

\begin{abstract}
Background: Current scientific guidelines have extended the indication for transcatheter aortic valve replacement (TAVR) to patients who present an intermediate risk for surgery and have been so far considered for conventional surgery. We previously demonstrated that the TAVR procedure generated profits despite elevated costs, but comparison with surgery has not been performed. The objective of this study was to assess the profitability of the TAVR procedure compared with conventional surgery in a high-volume French hospital.

Consecutive patients eligible for transfemoral TAVR or surgical aortic valve replacement (SAVR) were included retrospectively in this single-centre study between September 2014 and December 2015. The primary endpoint was the profitability of each procedure (defined as the ratio between the profit and total revenues), calculated for each patient. Secondary composite endpoints included major adverse events in the 30 days following procedure and breakdown of costs.
\end{abstract}

Results: Two hundred and thirty-eight patients were included in the TAVR group and 341 in the SAVR group. TAVR patients presented higher operative risk scores and more comorbidities. Compared with SAVR, TAVR was associated with higher profits ( $€ 2732 \pm 1768$ per patient vs. $€ 2177 \pm 2437$ per patient, $P<0.001)$ but also higher costs $(€ 27,778 \pm$ 4961 vs. $€ 17,813 \pm 6071, P<0.001)$ resulting in lower profitability $(9.3 \pm 5.7 \%$ vs. $11.7 \pm 10.1 \%, P<0.001)$. The price of the bioprosthesis represented $70 \%$ of the TAVR total cost.

Conclusions: TAVR performed in carefully selected patients was associated with higher profits than SAVR, but also higher costs resulting in lower profitability.

Keywords: Economical/cost-effectiveness, Aortic valve disease, percutaneous intervention, Cardiovascular diseases

\section{Introduction}

Transcatheter aortic valve replacement (TAVR) has revolutionised the prognosis of patients who present with severe aortic valve stenosis and cannot undergo conventional surgery. The non-inferiority of TAVR versus surgical aortic valve replacement (SAVR) has been demonstrated in high-risk patients [1], and recent studies are widening its use to patients with high-to-intermediate risk [2]. Current

\footnotetext{
* Correspondence: dr.fhuchet@gmail.com

${ }^{1}$ Service de Cardiologie, Hôpital Nord Laennec, Unité d'Hémodynamique et Cardiologie Interventionnelle, CHU de Nantes, Boulevard Professeur Jacques Monod, 44800 Saint-Herblain, France

Full list of author information is available at the end of the article
}

guidelines [3] support its use in symptomatic patients who are considered unsuitable for surgery as well as in intermediate risk patients with a favourable transfemoral access.

Consequently, the number of TAVR procedures is rapidly increasing. In 2016, in our centre, the implantation of percutaneous aortic bioprostheses became almost as frequent as the implantation of conventional isolated surgical bioprostheses, leading to rising costs and hospitalisations. We previously reported the elevated costs of the TAVR procedure [4], which are associated with large benefits for the institution. However, no direct comparison of cost-revenue has been performed between TAVR 
and conventional SAVR. TAVR prostheses are very expensive, and their implantation as a first-choice strategy in low-to-intermediate risk patients may be questionable in an era when healthcare costs are becoming a major concern. In this context, a comparative analysis could support the choice of the ideal strategy in patients eligible for both TAVR and SAVR.

Therefore, the objective of this study was to perform a comparative cost-revenue analysis of the TAVR and SAVR procedures in real-world patients managed in a high-volume French hospital.

\section{Methods}

\section{Study population}

Eligible patients were those scheduled to undergo transfemoral TAVR or SAVR using bioprostheses at the University Hospital of Nantes, France. The therapeutic choice was performed after an exhaustive preoperative appraisal by the heart team, in accordance with the European Society of Cardiology guidelines [5]. All consecutive eligible patients identified between September 2014 and December 2015 were included retrospectively into the study. Clinical data were collected in our local database. Data from TAVR patients were exported to the FRANCE-TAVI registry (managed by the French Society of Cardiology). All patients gave consent to the use of their data.

\section{Interventions and early follow-up}

Transfemoral TAVR procedures was performed using the balloon-expandable SAPIEN $3^{\circ}$ valve (Edwards Lifesciences, Irvine, CA, USA) or the self-expendable COREVALVE EVOLUT-R ${ }^{\circ}$ valve (Medtronic, Minneapolis, Minnesota, USA). The procedures were realised by two senior interventional cardiologists, as described previously [6]. The implantation of the percutaneous valves was systematically preceded by balloon dilatation of the native aortic valve. No pre-dilatation was performed in valve-in-valve procedures. A single dose of heparin (0.5 $\mathrm{mg} / \mathrm{kg}$ ) was injected immediately after positioning the major transarterial access, with no control of activated clotting time.

SAVR procedures were performed by a senior heart surgeon, with the assistance of a resident. The operating techniques and use of cardioplaegia were decided by the operating surgeon, but a surgical approach via full median sternotomy was systematically used. After surgery, patients were carefully monitored in intensive care unit (ICU) for a few hours, and then in a conventional unit prior to hospital discharge.

The antithrombotic regimen was delivered in accordance with concurrent guidelines [5]. Echocardiography was performed regularly during hospitalisation and before hospital discharge.

\section{Cost-revenue analysis}

Medicoeconomic data were collected from the French National Health Society and from the Medical Programme of Information Systems (PMSI: Programme de médicalisation des systèmes d'information).

For each patient, we calculated the total cost of the procedure (TAVR or SAVR), and compared it with the reimbursement received by the hospital. The costs were assessed using the 2014 French National Scale of Health Costs (available online http://www.scansante.fr/applications/enc-mco) and were dependent on length of hospital stay, development of early complications and requirement for a permanent pacemaker after the TAVR.

Four different costs were added to provide a total cost per patient: the clinical cost, which included all costs related to the hospitalisation unit (ICU and/or standard unit); the medicotechnical cost, which included all costs related to the invasive procedure, pacemaker implantation (if performed) and standard laboratory and imaging tests (this cost also included the remuneration of doctors and nurses during the procedure, and the maintenance and depreciation costs of the equipment); the logistical cost, which included medical (pharmacy, hygiene) and general (laundry, nourishment, administration, patient handling) logistical costs; and the direct cost, which included the price of the device(s), medicines and consumables.

The cost of medical devices and blood products and the remuneration of doctors and nurses during the procedure were estimated for each patient using a microcosting analysis. The total revenue for the hospital were determined for each patient as the sum of three separate revenues: a standard reimbursement defined by the patient's severity level; a supplement for patients monitored in an ICU or reanimation unit; and a reimbursement of implanted devices (in the TAVR group).

\section{Endpoints}

The primary endpoint was the profitability associated with the TAVR and SAVR procedures. We estimated the profit by calculating the difference between costs and revenues associated with each patient. Profitability was then calculated as the ratio between profit and total revenues for each patient.

The economic secondary endpoint was the breakdown of costs (clinical, medicotechnical, logistical and direct costs) for each treatment option.

The clinical secondary endpoints were major adverse events within 30 days of the procedure, defined according to the Valve Academic Research Consortium (VARC)-2 criteria [7], and including major vascular complications, major/life-threatening bleeding, major adverse postoperative events (defined as stroke and/or myocardial infarction), early rehospitalisation for heart 
failure, pacemaker requirement, tamponade and stage 3 acute kidney injury, analysed as separate endpoints.

\section{Subgroup analysis}

In the TAVR group, two subgroups were considered depending on the prostheses being implanted: SAPIEN 3 vs. COREVALVE. In the SAVR group, two subgroups were determined based on need for invasive cardiac monitoring (ICM).

\section{Statistical analysis}

Continuous variables are reported using means \pm standard deviations and were compared using Student's $t$ test. We used the $X^{2}$ test or Fisher's exact test to compare categorical variables. A two-sided $P$ value $<0.05$ was considered to indicate statistical significance. Statistical analyses were performed with $\mathrm{SPSS}^{\circ}$ software, version 20.0 (IBM Corp., Armonk, NY, USA).

\section{Results}

\section{Study population}

Between September 2014 and December 2015, the heart team prescribed transfemoral TAVR in 238 patients and SAVR 341 patients. The procedure was urgent in 15 TAVR patients $(6.3 \%)$ and in 14 SAVR patients $(4.1 \%$; $P=0.23)$

\section{Baseline characteristics}

Demographic and preoperative characteristics are presented in Table 1. Patients undergoing TAVR were significantly older than those selected for SAVR $(81.8 \pm 7.2$ years vs. $74.5 \pm 7.5$ years; $P<0.001$ ). Fewer men were present in the TAVR group ( $49 \%$ vs $59 \%, P=0.02$ ). Comorbidities were more frequent, and symptoms were more severe in the TAVR group, resulting in significantly higher operative risk scores.

\section{Procedure outcomes and length of stay}

In the TAVR group, 189 patients received a SAPIEN 3 prosthesis and 49 patients received a COREVALVE prosthesis. No switch to surgical procedure was necessary and a large majority of patients were operated under local anaesthesia $(214 / 238,90 \%)$. In the SAVR group, 101 patients out of 341 required ICM. Procedural success was high in both groups $(237 / 238$ in the TAVR group vs. 341/341 in the SAVR group). One severe aortic regurgitation was observed in the TAVR group (none in the SAVR group). Three patients (1.2\%) died within 30 days in the TAVR group vs. four $(1.2 \%)$ in the SAVR group $(P=0.99)$. The cause of death in the TAVR group was cardiac for one patient (annulus rupture during TAVR) and non-cardiac for two patients (both occurring after hospital discharge). The cause of death in the SAVR group was cardiac for three patients. No significant difference was observed between groups regarding the length of stay in ICU, but the total length of stay in hospital was shorter in the TAVR group $(9.0 \pm 6.0$ days vs. $11.7 \pm 5.5$ days; $P<0.001)$. No significant difference was observed regarding the main echocardiographic results following the procedure.

\section{Primary endpoint: Profitability analysis}

The profits associated with the TAVR and SAVR procedures are presented in Fig. 1. The profit observed for the

Table 1 Demographic and preoperative characteristics

\begin{tabular}{|c|c|c|c|}
\hline & $\operatorname{SAVR}(N=341)$ & $\operatorname{TAVR}(N=238)$ & $P$-values \\
\hline Male sex & $201(59 \%)$ & $118(49 \%)$ & 0.02 \\
\hline Age, years & $74.5 \pm 7.5$ & $81.8 \pm 7.2$ & $<0.001$ \\
\hline NYHA functional class I/II/II/IV & $57 / 217 / 57 / 10$ & 10/129/73/26 & $<0.001$ \\
\hline Logistic Euroscore, \% & $6.9 \pm 4.9$ & $14.7 \pm 9.4$ & $<0.001$ \\
\hline Euroscore II, \% & $2.3 \pm 2.3$ & $4.8 \pm 3.6$ & $<0.001$ \\
\hline Supraventricular arrhythmia & $32(9 \%)$ & $89(37 \%)$ & $<0.001$ \\
\hline Coronary artery disease & $76(22 \%)$ & $85(36 \%)$ & $<0.001$ \\
\hline Peripheral vascular disease & $24(7 \%)$ & $50(21 \%)$ & $<0.001$ \\
\hline Chronic obstructive pulmonary disease & $16(5 \%)$ & $54(23 \%)$ & $<0.001$ \\
\hline Permanent pacemaker & $4(1 \%)$ & $21(9 \%)$ & $<0.001$ \\
\hline Chronic kidney disease & $18(5 \%)$ & $116(49 \%)$ & $<0.001$ \\
\hline Liver disease & $1(0.3 \%)$ & $12(5 \%)$ & $<0.001$ \\
\hline History of cancer & $11(3 \%)$ & $40(17 \%)$ & $<0.001$ \\
\hline LVEF, \% & $61.8 \pm 9.1$ & $56.3 \pm 12.3$ & $<0.001$ \\
\hline Mean transaortic gradient in aortic stenosis, $\mathrm{mmHg}$ & $55.2 \pm 13.0$ & $51.0 \pm 19.3$ & 0.005 \\
\hline
\end{tabular}

Values are expressed as number of patients (percentage) or mean \pm standard deviation 


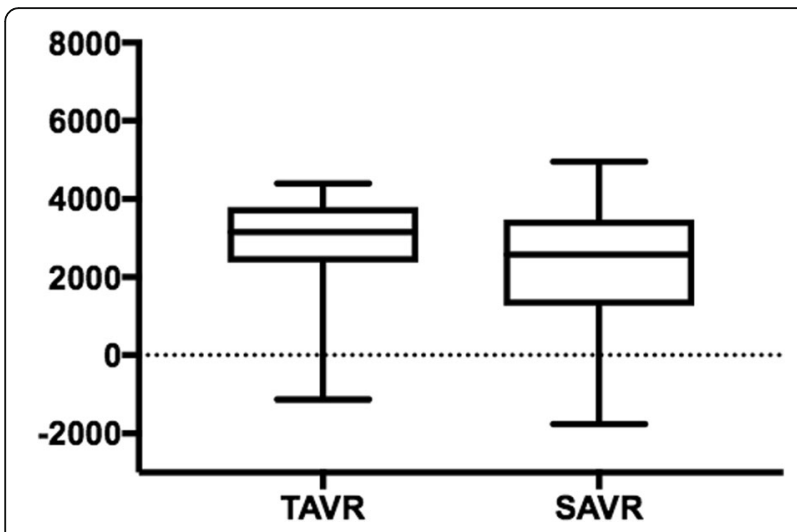

Fig. 1 Profit analysis, $€$ (median, quartiles, minimum and maximum) $P<0.001$. SAVR: surgical aortic valve replacement, TAVR: transcatheter aortic valve replacement

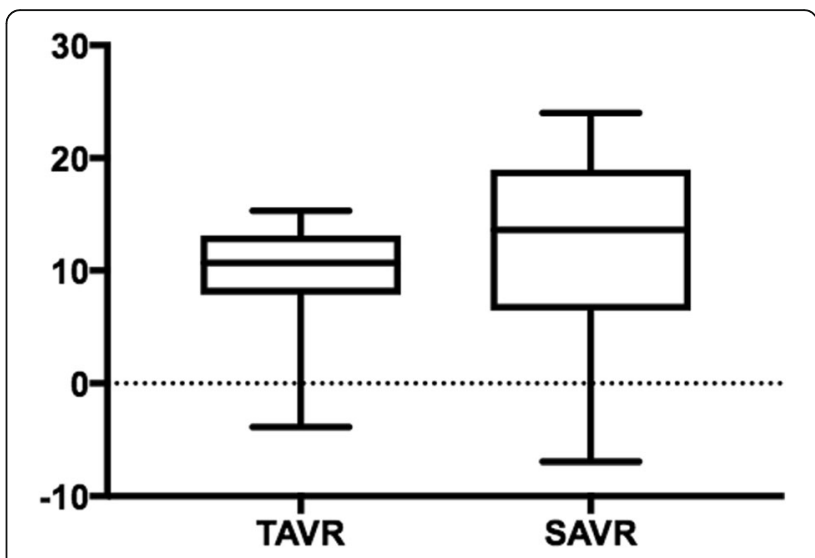

Fig. 2 Profitability of the procedure, \% (median, quartiles, minimum and maximum) $P<0.001$. SAVR: surgical aortic valve replacement, TAVR: transcatheter aortic valve replacement
TAVR group was significantly higher than for the SAVR group ( $€ 2732 \pm 1768$ vs $€ 2177 \pm 2437, P=0.002)$. The mean costs per patient for the TAVR and SAVR procedures were $€ 27,778 \pm 4961$ and $€ 17,813 \pm 6071$, and the mean revenues per patient were $€ 30,509 \pm 3760$ and $€ 19,989 \pm 4739$ (all $P<0.001$ ), respectively. Thus, the TAVR procedure presented an additional cost of $€ 9965$ (56\%) per patient compared with the SAVR procedure, resulting in a reduced profitability $(9.3 \pm 5.7 \%$ vs. $11.7 \pm$ 10.1\%, $P<0.001$, Fig. 2).

\section{Secondary economic endpoint: Breakdown of costs}

The breakdown of costs is presented in Fig. 3. Significant differences were observed between the TAVR and SAVR groups regarding all considered costs (all $p<0.02$ ). All costs were significantly lower for TAVR patients, except for direct costs, which were mostly driven by the price of the bioprosthesis representing $70 \%$ of the total cost and $87 \%$ of device-related costs. Logistical and medicotechnical costs contributed less than $12 \%$ to the total costs in the TAVR group, whereas they represented almost $40 \%$ in the SAVR group.

\section{Secondary clinical endpoint: Major adverse events}

Major adverse events within 30 days of the procedure are presented in Table 2. Patients in the TAVR group required more frequently a permanent pacemaker implantation $(17 \%$ vs. $3 \%, P<0.001)$, and presented higher rates of disabling stroke or major vascular complications. SAVR patients required more frequently blood transfusions and prolonged mechanical ventilation.

\section{Subgroup analysis}

In the TAVR group, the patients receiving a SAPIEN 3 prosthesis $(n=189)$ and those receiving a COREVALVE prosthesis $(n=49)$ had similar baseline characteristics (not shown), except for a more frequent prior aortic bioprosthesis implantation in the COREVALVE subgroup, due to our team preferring the implantation of a self-expandable prosthesis for the valve-in-valve procedures. No significant difference was observed between subgroups regarding the primary endpoint (profitability $9.4 \pm 5.9 \%$ in the SAPIEN 3 patients vs. $8.8 \pm 4.8 \%$ in the COREVALVE patients, $P=0.45$ ) and the occurrence of major adverse events. The analysis of the breakdown of costs demonstrated significantly higher direct costs in the COREVALVE subgroup ( $€ 23,351 \pm 5887$ vs. $€ 22,189$ $\pm 1617 ; \quad P=0.02$ ), caused by significantly higher device-related costs $(€ 21,975 \pm 5653$ vs. $€ 20,881 \pm 1518$; $P=0.02$ ). No significant difference was observed regarding the requirement for a permanent pacemaker in the patients not implanted previously to the valvular procedure (33 patients out of 170 in the SAPIEN 3 subgroup vs. 7 out of 30 in the COREVALVE subgroup, $P=0.49$ ).

In the SAVR group, the patients who required ICM following the surgery $(n=101)$ presented more frequent comorbidities. They also had a more severe New-York Heart Association (NYHA) functional class and a significantly longer hospital stay. The profitability associated with the procedure was significantly higher in the subgroup of patients who required ICM $(14.9 \pm 10.2 \%$ vs. $11.2 \pm 8.7, P<0.001)$, in line with higher hospital revenues $(€ 22,879 \pm 4779$ vs $€ 18,773 \pm 3401, P<0.001)$ and despite significantly higher costs $(€ 19,732 \pm 6275$ vs. $€ 17,005 \pm 4528, P<0.001$ ),

\section{Discussion}

Our study demonstrates that the TAVR procedure performed with the new generation of percutaneous prostheses is associated with higher profits for the hospital than conventional surgery, but it also 


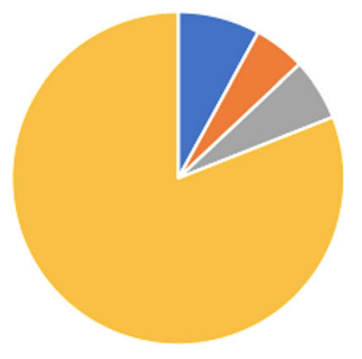

TAVR

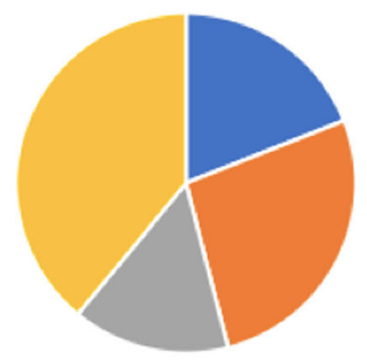

SAVR

Fig. 3 Overall breakdown of costs. SAVR: surgical aortic valve replacement, TAVR: transcatheter aortic valve replacement

involves significantly higher costs, which results in lower profitability. Our cohort included real-world patients recruited in a high-volume French centre and the results describe the current relative economic status of both procedures in the context of the French healthcare system.

The study population was representative of the patients seen in current practice. In the TAVR group, despite old age and comorbidities, most patients presented intermediate operative risk scores, as a result of careful and multidisciplinary selection [8,9], whereas in the SAVR group, a large majority of patients had low operative risk scores. In line with recent studies and registries $[10,11]$, procedural success was high in both groups (237/238 in the TAVR group vs. $341 / 341$ in the SAVR group). Seven patients (1.2\%) died within 1 month of the procedure.

The percutaneous implantation of an aortic bioprosthesis has been associated with a reduction in hospital length of stay compared with conventional surgery, especially in elderly patients with comorbidities and patients who have suitable transfemoral access [12]. This observation was confirmed in our study, where TAVR patients had a significantly shorter hospital stay despite old age and numerous comorbidities. Some studies have considered the possibility of discharging patients within a few days of a TAVR procedure $[13,14]$. This may minimally increase the profit generated from the procedure as the clinical costs only accounted for $9 \%$ of the total TAVR costs. On the other hand, the length of hospital stay following conventional surgery would be more complicated to shorten, and will continue to contribute significantly to the clinical costs, which account for $20 \%$ of the total SAVR costs.

Medicoeconomic data on TAVR and comparisons with SAVR are limited because of the broad diversity among national healthcare systems. Mitigated results regarding the balance between health costs and quality of life measured as quality-adjusted life years (QALY) have been published on the populations enrolled in the PARTNER studies from the US [12] and Canadian [15] perspectives. Transfemoral TAVR was found more cost-effective than conventional surgery, but the observed difference was marginal. Moreover, Tam et al. [15] reported that the small improvement in QALY observed with TAVR was counterbalanced by an increase in total lifetime costs estimated to around CAD 10,000 per patient. In this context of slight benefits associated with important

Table 2 Major adverse events within 30 days of the procedure

\begin{tabular}{llll}
\hline & SAVR $(N=341)$ & TAVR $(N=238)$ & $P$-values \\
\hline Disabling stroke & $0(0 \%)$ & $4(2 \%)$ & 0.03 \\
Myocardial infarction & $2(0.6 \%)$ & $2(0.8 \%)$ & 0.70 \\
Major vascular complications & $0(0 \%)$ & $12(5 \%)$ & $<0.001$ \\
Blood transfusion requirement & $43(13 \%)$ & $15(6 \%)$ & 0.02 \\
Respiratory failure requiring prolonged ventilation & $30(9 \%)$ & $2(0.8 \%)$ & $40(17 \%)$ \\
Pacemaker requirement & $9(3 \%)$ & $4(2 \%)$ & $<0.001$ \\
Stage 3 acute kidney injury & $4(1 \%)$ & $1(0.4 \%)$ & 0.72 \\
Dialysis requirement & $4(1 \%)$ & $6(3 \%)$ & 0.65 \\
Early rehospitalization for heart failure & $2(0.6 \%)$ & 0.07 \\
\hline Val
\end{tabular}

Values are expressed as number of patients (percentage)

SAVR surgical aortic valve replacement, TAVR transcatheter aortic valve replacement 
increases in costs, it seems difficult to broaden the TAVR procedure to low risk patients.

To our point of view, as long as the TAVR remain that expensive, the specific associated issues should be solved before enlarging the indication. These include uncertainty on bioprosthesis durability and choice of the optimal antithrombotic regimen (especially in the context of concern regarding prostheses thromboses) as well as the frequent requirement for permanent pacemaker implantation. On the other hand, a large part of the TAVR overall cost is attributed to the price of the bioprosthesis and the implantation of a pacemaker further increases the procedural cost. We believe a reduction in the total cost of the TAVR procedure would mostly be achieved by significantly reducing the price of the device and limiting the need for pacemaker implantation.

The subgroup analysis comparing procedural costs with the SAPIEN 3 and COREVALVE prostheses demonstrated significantly higher direct costs and a trend for higher total costs with the COREVALVE prosthesis. The COREVALVE prosthesis implantation has been associated with a moderate increased risk of pacemaker implantation compared with balloon-expandable valve replacement [16]. Yet, in our small subgroup analysis, pacemaker requirement rates were not significantly different between TAVR subgroups and could not explain the increase in device-related costs observed in the COREVALVE subgroup. The increase in device-related costs were probably related to the requirement of a separate introducer, a separate balloon for the predilatation of the native aortic valve, and more frequent necessity of post-dilatation in the self-expendable subgroup.

In the surgical group, the requirement for ICM was associated with significantly higher profits, which further emphasises the low profit to be derived from conventional surgery in the absence of ICM. Moreover, patients requiring ICM are likely to have higher operative risk and become candidates for a future TAVR procedure.

\section{Study limitations}

We produced a single-centre study, but this limitation was balanced by high volume and use of standard patient recruitment. The length of hospital stay was long in both groups during the study time, contributing to limit the profitability of both techniques. Regardless of these limitations, both techniques were found to be highly profitable for the hospital. Finally, the price of the TAVR devices recently decreased to $€ 14,283$ (application date: August 2018). This will reduce the cost of the TAVR procedure that still remains more expensive than the SAVR procedure but could demonstrate higher profitability in a near future.

\section{Conclusion}

This study demonstrates that TAVR generates slightly higher profits than SAVR in an academic high-volume hospital. However, total costs are significantly increased for a TAVR procedure resulting in lower profitability. A large part of the TAVR cost is related to the price of the bioprosthesis. If the indication for TAVR is to be extended to patients with low operative risk, technical issues such as pacemaker requirement will have to be addressed and the price of the device will have to decrease.

\section{Abbreviations \\ ICM: Invasive cardiac monitoring; ICU: Intensive care unit; PMSI: Medical Programme of Information Systems (Programme de médicalisation des systèmes d'information); QALY: Quality-adjusted life years; SAVR: Surgical aortic valve replacement; TAVR: Transcatheter aortic valve replacement}

\section{Acknowledgements}

Not applicable.

\section{Funding}

Not applicable,

\section{Availability of data and materials}

All data may be discussed with the corresponding author

(dr.fhuchet@gmail.com) as well as the methods, using email request.

The economic data were collected using the national French program of medical information.

The clinical data were collected in a local database and secondary exported to the French national registry of percutaneous aortic valves (FRANCE-TAVI registry).

\section{Authors' contributions}

All authors provided separated parts of the data. All of them read, corrected and approved the submitted manuscript. Fd'A and GG provided economic data. TS and JCR performed cardiac surgery and provided the surgical data. VL, JP, TM and PG performed percutaneous aortic valve replacements, and provided percutaneous patients' clinical data. TM initiated and directed the study. FH and JC-P created the database and wrote the manuscript. FH performed the statistical analysis.

\section{Competing interests}

The authors declare that they have no competing interests.

\section{Publisher's Note}

Springer Nature remains neutral with regard to jurisdictional claims in published maps and institutional affiliations.

\section{Author details}

'Service de Cardiologie, Hôpital Nord Laennec, Unité d'Hémodynamique et Cardiologie Interventionnelle, CHU de Nantes, Boulevard Professeur Jacques Monod, 44800 Saint-Herblain, France. ${ }^{2}$ Pharmacie Centrale, Hôpital Saint-Jacques, CHU de Nantes, 44093 Nantes, France. ${ }^{3}$ Service de chirurgie cardio-thoracique, Hôpital Nord Laennec, CHU de Nantes, 44800

Saint-Herblain, France.

Received: 17 October 2018 Accepted: 7 February 2019

Published online: 14 February 2019

\section{References}

1. Smith CR, Leon MB, Mack MJ, et al. Transcatheter versus surgical aortic-valve replacement in high-risk patients. N Engl J Med. 2011;364:2187-98.

2. Leon MB, Smith CR, Mack MJ, et al. Transcatheter or surgical aortic-valve replacement in intermediate-risk patients. N Engl J Med. 2016;374:1609-20.

3. Baumgartner H, Falk V, Bax JJ, et al. 2017 ESC/EACTS guidelines for the management of valvular heart disease. Eur Heart J. 2017;38:2739-91. 
4. Huchet F, d'Acremont $F$, Letocart $V$, et al. Is transcatheter aortic valve replacement a profitable procedure in a high-volume French hospital? Arch Cardiovasc Dis. 2018.

5. Vahanian $\mathrm{A}$, Alfieri $\mathrm{O}$, Andreotti $\mathrm{F}$, et al. Guidelines on the management of valvular heart disease (version 2012): the joint task force on the management of Valvular heart disease of the European Society of Cardiology (ESC) and the European Association for Cardio-Thoracic Surgery (EACTS). Eur J Cardiothorac Surg. 2012:42:S1-44.

6. Eltchaninoff H, Zajarias A, Tron C, et al. Transcatheter aortic valve implantation: technical aspects, results and indications. Arch Cardiovasc Dis. 2008;101:126-32.

7. Kappetein AP, Head SJ, Généreux P, et al. Updated standardized endpoint definitions for transcatheter aortic valve implantation: the Valve Academic Research Consortium-2 consensus document. J Am Coll Cardiol. 2012;60: 1438-54.

8. Ruparelia N, Latib A, Buzzatti N, et al. Long-term outcomes after Transcatheter aortic valve implantation from a single high-volume center (the Milan experience). Am J Cardiol. 2016;117:813-9.

9. Levi A, Landes U, Assali AR, et al. Long-term outcomes of 560 consecutive patients treated with Transcatheter aortic valve implantation and propensity score-matched analysis of early- versus new-generation valves. Am J Cardiol. 2017:119:1821-31.

10. Wendler O, Schymik G, Treede H, et al. SOURCE 3 registry: design and 30day results of the European Postapproval registry of the latest generation of the SAPIEN 3 Transcatheter heart valve. Circulation. 2017;135:1123-32.

11. Wendler O, Schymik G, Treede H, et al. SOURCE 3: 1-year outcomes posttranscatheter aortic valve implantation using the latest generation of the balloon-expandable transcatheter heart valve. Eur Heart J. 2017;38:2717-26.

12. Reynolds MR, Magnuson EA, Lei Y, et al. Cost-effectiveness of transcatheter aortic valve replacement compared with surgical aortic valve replacement in high-risk patients with severe aortic stenosis: results of the PARTNER (placement of aortic Transcatheter valves) trial (cohort a). J Am Coll Cardiol. 2012:60:2683-92.

13. Durand $E$, Eltchaninoff $H$, Canville $A$, et al. Feasibility and safety of early discharge after transfemoral transcatheter aortic valve implantation with the Edwards SAPIEN-XT prosthesis. Am J Cardiol. 2015;115:1116-22.

14. Sud M, Qui F, Austin PC, et al. Short length of stay after elective Transfemoral Transcatheter aortic valve replacement is not associated with increased early or late readmission risk. J Am Heart Assoc. 2017;6.

15. Tam DY, Hughes A, Fremes SE, et al. A cost-utility analysis of transcatheter versus surgical aortic valve replacement for the treatment of aortic stenosis in the population with intermediate surgical risk. J Thorac Cardiovasc Surg. 2018;155:1978-88 e1.

16. Siontis GC, Juni P, Pilgrim T, et al. Predictors of permanent pacemaker implantation in patients with severe aortic stenosis undergoing TAVR: a meta-analysis. J Am Coll Cardiol. 2014;64:129-40.

Ready to submit your research? Choose BMC and benefit from:

- fast, convenient online submission

- thorough peer review by experienced researchers in your field

- rapid publication on acceptance

- support for research data, including large and complex data types

- gold Open Access which fosters wider collaboration and increased citations

- maximum visibility for your research: over $100 \mathrm{M}$ website views per year

At $\mathrm{BMC}$, research is always in progress.

Learn more biomedcentral.com/submissions 